Article

\title{
Suitability of the Cyclic Voltammetry Measurements and DPPH• Spectrophotometric Assay to Determine the Antioxidant Capacity of Food-Grade Oenological Tannins
}

\author{
Arianna Ricci ${ }^{1}$, Giuseppina Paola Parpinello ${ }^{1, *} \mathbb{C}$, Nemanja Teslić ${ }^{2}(\mathbb{D}$, \\ Paul Andrew Kilmartin ${ }^{3}$ (D) and Andrea Versari ${ }^{1}$ (D) \\ 1 Department of Agricultural and Food Sciences, Alma Mater Studiorum-University of Bologna, \\ Piazza Goidanich 60, 47521 Cesena, FC, Italy \\ 2 Institute of Food Technology, University of Novi Sad, Bulevar cara Lazara 1, 21000 Novi Sad, Serbia \\ 3 School of Chemical Sciences, The University of Auckland, Private Bag 92019, Auckland 1142, New Zealand \\ * Correspondence: giusi.parpinello@unibo.it; Tel.: +39-0547-338-118
}

Academic Editors: Jacek Namieśnik and Justyna Płotka-Wasylka

Received: 21 July 2019; Accepted: 12 August 2019; Published: 13 August 2019

\begin{abstract}
Twenty commercially available oenological tannins (including hydrolysable and condensed) were assessed for their antiradical/reducing activity, comparing two analytical approaches: The 2,2-diphenyl-1-picrylhydrazyl (DPPH•) radical scavenging spectrophotometric assay and the cyclic voltammetry (CV) electrochemical method. Electrochemical measurements were performed over a $-200 \mathrm{mV}-500 \mathrm{mV}$ scan range, and integrated anodic currents to $500 \mathrm{mV}$ were used to build a calibration graph with (+)-catechin as a reference standard (linear range: From 0.0078 to $1 \mathrm{mM}$, $\mathrm{R}^{2}=0.9887$ ). The CV results were compared with the DPPH• assay (expressed as \% of radical scavenged in time), showing high correlation due to the similarity of the chemical mechanisms underlying both methods involving polyphenolic compounds as reductants. Improved correlation was observed by increasing the incubation time with DPPH• to $24 \mathrm{~h}\left(\mathrm{R}^{2}=0.925\right)$, demonstrating that the spectrophotometric method requires a long-term incubation to complete the scavenging reaction when high-molecular weight tannins are involved; this constraint has been overcome by using instant $\mathrm{CV}$ measurements. We concluded that the CV represents a valid alternative to the DPPH• colorimetric assay, taking advantage of fast analysis and control on the experimental conditions and, because of these properties, it can assist the quality control along the supply chain.
\end{abstract}

Keywords: oenological tannins; antioxidant activity; 2,2-diphenyl-1-picrylhydrazyl; cyclic voltammetry; glassy carbon electrode

\section{Introduction}

The word 'tannins' designates a class of polyphenolic compounds characterized by macromolecules and high-molecular weight polymers (500 Da-2000 Da) including complex structures with variable degrees of polymerization, arranged through condensed and hydrolysable chemical bonds. Tannins are synthesized by plants as a defense against pathogens; in fact, they are good electron-hydrogen donors and protect vegetable tissues by neutralizing oxidative molecules (mainly peroxides and reactive oxygen species, ROS) which are formed under biological stress conditions. Due to these properties, tannins are considered natural valuable compounds and they can be isolated from vegetable tissues using suitable extraction methods in their technological exploitation [1,2].

According to their chemical structures, tannins can be classified into two major groups, condensed tannins or proanthocyanidins (the latter name refers to their ability to release anthocyanidins under 
mild temperature and acidic conditions), and hydrolysable tannins, which are polyesters of gallic, ellagic, and hexahydroxydiphenoyl acids. When extracts are obtained from the raw vegetal sources, classification could be performed according to their botanical origin [3,4].

Tannins from grapefruit, tea leaves, oak wood, chestnut wood, gallnuts, and tara are traditionally exploited in the wine industry; they are available as lyophilized powders or stabilized solutions and directly added to wines and musts as 'processing aids' for the precipitation of excess proteinaceous matter. Regardless of the role of tannins in oenology, which is covered by current regulations (OIV International Code of Oenological Practices, 3.2 Clarification of Wine), these compounds play a key role on the chemical stability and sensory properties of wine. The use of commercial formulations is recommended for several purposes, including clarification and microbiological stabilization of musts, chemical and color stabilization of wines, strengthening of light-bodied wines [5]. Among these, the antioxidant activity constitutes a challenging opportunity for the technological exploitation of tannins in oenology, to assist or replace the use of sulphur dioxide for preventing oxidative damage in wine $[6,7]$.

For a deeper insight on the reactivity of tannins we need to consider their composition and molecular structures, including the extent of the polymerization, substituents and steric hindrance, and the chemical reactivity involved.

From a chemical perspective, the antioxidant activity of polyphenols occurs by the neutralization of ROS, which can be generated in wines and musts in the presence of catalysts and/or catalytic conditions (Figure 1), through hydrogen (HT) or electron transfer mechanism (ET). Both transfer mechanisms are dependent on the experimental conditions, i.e., the $\mathrm{pH}$ value of the solution or the polarity of the medium $[8,9]$.
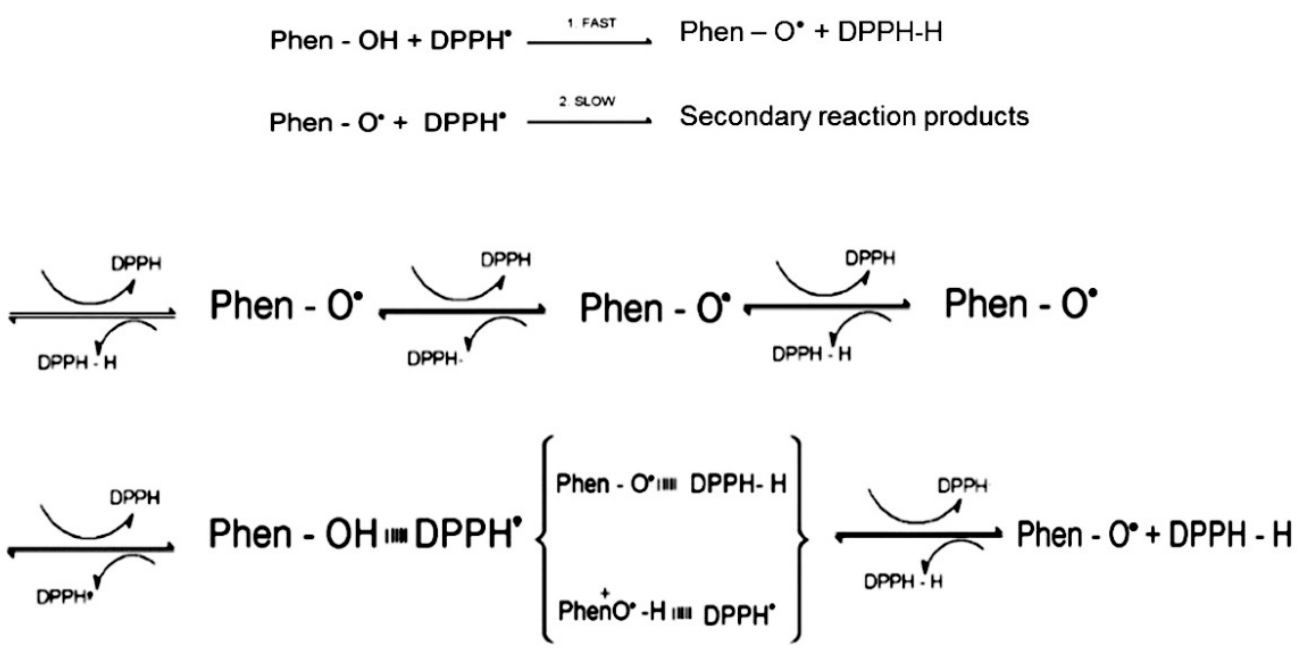

Figure 1. Schematic representation of the reaction between 2,2-diphenyl-1-picrylhydrazyl (DPPH)• radical and a generic polyphenolic compound (Phen-OH).

Targeted analytical assays have been developed to assess the antioxidant activity of polyphenolic compounds, providing a total antioxidant capacity (TAC) value in standard solutions and in real matrices. These assays are based on the ability of antioxidants to reduce $\mathrm{Cu}(\mathrm{II})$ and $\mathrm{Fe}(\mathrm{III})$ transition metals, responsible for the catalytic initiation of the oxidative chain, or to scavenge radicals, which are responsible for the oxidation of organic substrates. Among these, the 2,2-diphenyl-1-picrylhydrazyl $(\mathrm{DPPH} \bullet)$ assay is based on the use of a stable synthetic radical in solution, with an unpaired valence electron at the nitrogen bridging atom. DPPH• is neutralized through the transfer of a hydrogen atom or an electron (or both, in sequence) made available by antioxidant polyphenols. The antioxidant activity of a sample is referenced against the scavenging activity of a standard reducing molecule at a known concentration (i.e., Trolox), or as a percentage of radical scavenging, corresponding to the lowering of the absorption peak of the radical species $(517 \mathrm{~nm})$ over time $[10,11]$. 
The DPPH• procedure involves incubation of the reaction mixture for a set, but variable, time period (from minutes to hours), before reading the decrease in absorbance at $517 \mathrm{~nm}$. However, it has been shown that under some experimental conditions this time duration is not sufficient to reach the steady state. The kinetics of radical scavenging is very fast in the first step with many polyphenols, but the reactive polyphenol (Phen-O•) obtained after HT or ET processes can undergo further reactions, influencing the overall stoichiometry (i.e., the number of radical molecules reduced by a single molecule of reductant). Previous studies have demonstrated that a slight but continuous decrease in absorbance could still be observed up to $24 \mathrm{~h}$ of incubation $[12,13]$. The stoichiometry of the overall reaction is strongly influenced by the fate of radical species formed after reduction of DPPH•. The incubation time can be lowered through sample dilution, but this might complicate the assay procedure, requiring time-consuming trials in order to determine the optimum dilution conditions. The ratio of the effective content of active polyphenols to the actual weight of the extract powder is highly variable among different commercial formulations, so that a standard dilution value cannot be applied.

The purpose of this work is to evaluate the results of DPPH• measurements on oenological tannin solutions prepared at a standard powder weight, and to compare these results with the CV method, considered as a potential alternative to the controversial spectrophotometric method, affected by the variable time needed to run the assay and possible inferences due to its application in coloured samples. The main oenological tannins and their basic monomeric fractions show redox properties that were already elucidated in previous works $[7,14,15]$. Gallic acid, (+)-catechin and (-)-epicatechin moieties, exhibit low oxidation potentials among polyphenols due to the ortho-diphenol substitution of their aromatic rings. These molecular features give a first oxidation peak close to $400 \mathrm{mV}$ (compared to $\mathrm{Ag} / \mathrm{AgCl})$ in a model wine solution $[14,16]$.

It is reasonable to measure the charge passed to $500 \mathrm{mV}$ (integrated peak of the voltammogram), obtaining a parameter comparable to the DPPH• response. This is due to the low oxidation potential of the synthetic DPPH• radical itself, which exhibits a reversible redox couple at about $100 \mathrm{mV}$ more positive than catechol-containing polyphenols; the DPPH• radical can oxidize catechol, pyrogallol and related moieties, but is less effective in reducing benzoic acids, some hydroxycinnamic acids like coumaric acid, or flavonoid A-ring polyphenols. A scan to potentials higher than $500 \mathrm{mV}$ would capture the latter phenolic groups that do not provide significant contribution to the DPPH• radical scavenging mechanism [17].

Previous correlations between DPPH• and electrochemical methods for determination of the antioxidant activity of polyphenols have been attempted so far with satisfactory results [7,18-20]. In the present work, commercial tannin powders were dissolved in a medium reproducing wine conditions (hydro-alcoholic buffer solution, $\mathrm{pH}$ 3.6) and were assessed using both $\mathrm{CV}$ and DPPH• methods. For the spectrophotometric method, different incubation times were evaluated in order to obtain steady-state conditions, and the time-dependent results were correlated with the integrated peak current provided by CV. The potential use of CV as a fast and easy-to-use analytical tool for monitoring the protective action of oenological additives was evaluated, as an alternative to the reliable but time-consuming DPPH• antioxidant assay which is routinely used in the laboratories.

\section{Results and Discussion}

\subsection{Cyclic Voltammetry}

The electrochemical and redox properties of tannins were investigated by analyzing the voltammetry profiles. Voltammograms of representative commercial extracts recorded over a wide range of potentials ( $-200 \mathrm{mV}$ to $1000 \mathrm{mV}$ ) are shown in Figure 2, along with the relevant monomer which is expected to contribute to the polymeric structure, according to the chemical class (Figure 2a: Proanthocyanidin/(+)-catechin; Figure 2b: Ellagitannin/ellagic acid; Figure 2c: Gallotannin/gallic acid). Despite the fact that the whole peak area was used to determine the redox activity, the maximum of electrochemical peaks was considered suitable for a qualitative analysis and to attempt identification 
based on the botanical sources. The main redox peaks, which are listed in Table 1, are almost overlapping in the potential range up to $500 \mathrm{mV}$, which is the range of interest for the most active molecular structures, in agreement with electrochemical parameters which were previously reported in the literature for polyphenolic monomers [14,21].
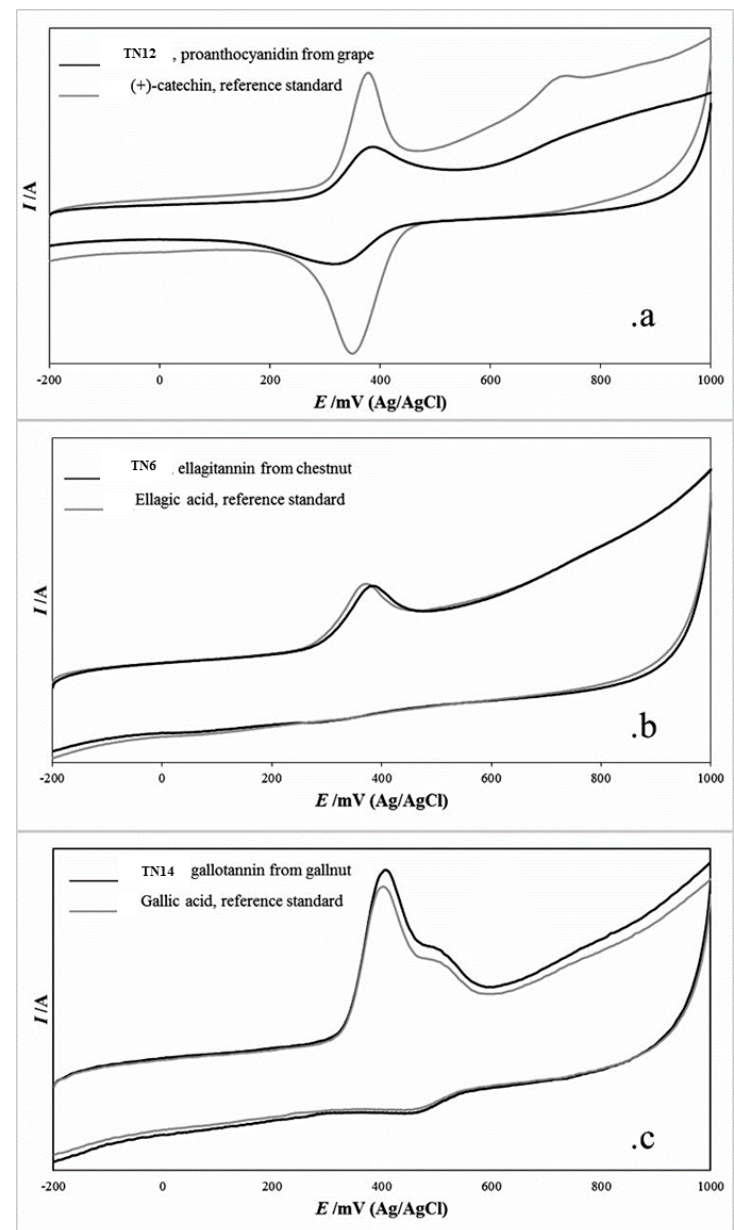

Figure 2. Cyclic voltammograms (background subtracted) for the couples: proanthocyanidin TN12/(+) - catechin; (a) ellagitannin TN6/ellagic acid; (b) gallotannin TN14/gallic acid; (c) in the model wine solution, measured at $100 \mathrm{mV} / \mathrm{s}$ above the potential range: $-200 \mathrm{mV}$ to $1000 \mathrm{mV}$, at a $3 \mathrm{~mm}$ glassy carbon electrode.

Table 1. Anodic $\left(E_{p, a}\right)$ and cathodic $\left(E_{p, c}\right)$ peak potentials obtained by scanning tannin solutions in the range of $-200 \mathrm{mV}$ to $500 \mathrm{mV}$. The redox couples obtained by reversible processes are labelled in italics. A detailed description of tannin samples (TNs) according to the information by suppliers is provided in Table 4 of the 'Materials and Methods' section.

\begin{tabular}{ccccc}
\hline \multirow{2}{*}{ Samples } & \multicolumn{3}{c}{ E (mV versus Ag/AgCl) } \\
\cline { 2 - 6 } & \multicolumn{2}{c}{$\mathbf{E}_{\mathbf{p}, \mathbf{a}}$} & \multicolumn{2}{c}{$\mathbf{E}_{\mathbf{p}, \mathbf{c}}$} \\
\hline (+)-Catechin, std & - & 389 & - & 354 \\
Ellagic acid, std & - & 367 & - & - \\
Gallic acid, std & - & 391 & - & - \\
TN1 & - & 402 & - & - \\
TN2 & 313 & 419 & 294 & 391 \\
TN3 & 290 & 383 & - & 329 \\
TN4 & - & 406 & - & - \\
TN5 & 308 & 406 & - & 337 \\
\hline
\end{tabular}


Table 1. Cont.

\begin{tabular}{|c|c|c|c|c|}
\hline \multirow{3}{*}{$\begin{array}{c}\text { Samples } \\
\text { TN6 }\end{array}$} & \multicolumn{4}{|c|}{$\mathrm{E}(\mathrm{mV}$ versus $\mathrm{Ag} / \mathrm{AgCl})$} \\
\hline & \multicolumn{2}{|c|}{$\mathrm{E}_{\mathrm{p}, \mathrm{a}}$} & \multicolumn{2}{|c|}{$E_{p, c}$} \\
\hline & - & 384 & - & - \\
\hline TN7 & - & 399 & - & 338 \\
\hline TN8 & 290 & 399 & - & 332 \\
\hline TN9 & - & 404 & - & - \\
\hline TN10 & - & 386 & - & - \\
\hline TN11 & - & 380 & - & - \\
\hline TN12 & - & 399 & - & 321 \\
\hline TN13 & - & 401 & - & - \\
\hline TN14 & - & 413 & - & 395 \\
\hline TN15 & - & 379 & - & - \\
\hline TN16 & - & 370 & - & - \\
\hline TN17 & - & 382 & - & - \\
\hline TN18 & - & 384 & - & 328 \\
\hline TN19 & - & 396 & - & - \\
\hline TN20 & - & 404 & - & - \\
\hline
\end{tabular}

When considering pure condensed tannins (proanthocyanidins), the position of the electrochemical peaks matched with the redox couple found in the (+)-catechin standard. TN2, a tannin extracted from green tea leaves, showed an occasional early oxidation peak at $313 \mathrm{mV}$ with reversible cathodic peak $\left(E_{p, c}\right)$ at $294 \mathrm{mV}$; this electrochemical feature was not observed in the other proanthocyanidins of the series, and could be tentatively attributed to the peculiar composition in the galloylated and galloyl/gallate flavanols expected in tea leaves extracts [22-24]. The main redox couple confirmed the voltammetry profile expected for condensed tannins-based extracts, with an anodic peak at $419 \mathrm{mV}$ related to a highly reversible process $\left(E_{p, a}-E_{p, c}=28 \mathrm{mV}\right)$.

The presence of a prominent, reverse cathodic peak, which is clearly observed in samples TN7 and TN12, which are grape-derived extracts, was related to the occurrence of the redox couple ortho-diphenol/quinone formed during the oxidation process; this can be considered an electrochemically reversible process, despite the peak separation values of the redox couple composed by the anodic and cathodic peak, $\mathrm{E}_{\mathrm{p}, \mathrm{a}}-\mathrm{E}_{\mathrm{p}, \mathrm{c}}(\Delta \mathrm{E})$ found in most of the proanthocyanidins investigated were larger than the $29 \mathrm{mV}$ theoretical value expected for a fully reversible system. A value of $\Delta \mathrm{E}$ $=35 \mathrm{mV}$ was obtained for the (+)-catechin monomeric standard. The presence of highly oxidizable substrates with high reversibility, reflect the peculiar antioxidant capacity of proanthocyanidins, and will be discussed in more detail in Section 2.3.

$\mathrm{TN} 8$, is a blended formulation including ellagitannins and proanthocyanidins; the prevalence of (+)-catechin - related oxidation peaks among the overlapping electrochemical features confirm that the proanthocyanidins are prevailing in this formulation.

In the case of ellagitannins and gallotannins sourced from tara, gall, oak wood, chestnut, fruit tree wood, and their blended formulations, the anodic oxidation peaks were located in the range $370 \mathrm{mV}-406 \mathrm{mV}$ and related to largely irreversible oxidation processes, as a confirmation of the electrochemical features observed in the ellagic acid and gallic acid monomers. Nevertheless, small amounts of flavanol compounds present in wood extracts and blended formulations (TN3, 5, 14, 18) could contribute to the small return cathodic peak observed.

A further sub-grouping was assigned for the anodic oxidation peaks around $370 \mathrm{mV}-386 \mathrm{mV}$ and $399 \mathrm{mV}-406 \mathrm{mV}$. The first grouping was attributed to the gallic and ellagic acid carboxylic moieties $(-\mathrm{COOH})$, which is largely involved in the antioxidant activity of these polyphenolic compounds and their derivatives; the second grouping may be related to the occurrence of esterification and/or coordination to central sugar moieties, which are recurring in hydrolysable extracts as previously disclosed by MALDI-TOF investigations [24,25]. 


\subsection{Practical Issues Related to the Use of DPPH• Assay to Determine Antiradical Activity of Tannins}

The DPPH• colorimetric assay is based on the ability of reducing molecules to scavenge the 2,2-diphenyl-1-picrylhydrazyl synthetic radical dissolved in an alcoholic solution, thus obtaining its neutral form. The process is monitored through the conversion of the violet-colored radical with the absorbance at $517 \mathrm{~nm}$ into a pale-yellow solution, and the antiradical activity was reported at a (\%) decrease of such absorbance at different incubation times: $15 \mathrm{~min}, 30 \mathrm{~min}, 60 \mathrm{~min}, 180 \mathrm{~min}$, and $24 \mathrm{~h}$. The overall kinetic curve describing the radical drop up to $24 \mathrm{~h}$ gained increasing complexity while increasing the incubation time; nevertheless, all tannins reported the same kinetic trend when considering the decrease in the $517 \mathrm{~nm}$ absorbance up to 60 min incubation, highly fitted with zero-order kinetic rates $\left(R^{2}>0.90\right)$. The partial kinetics corresponding to the primary, fast step of $\mathrm{DPPH} \bullet$ scavenging by tannins (see Figure 1) are reported in the Table 2.

Table 2. Zero-order kinetic rates of DPPH• radical scavenging by tannins over a 60-min incubation period.

\begin{tabular}{cc}
\hline Samples & $\begin{array}{r}\text { DPPH• Scavenging Kinetic Rates } \\
\text { [0-60 min Incubation] }\end{array}$ \\
\hline Code & \% decrease Abs $_{517} \mathrm{~nm} / \mathrm{min}$ \\
\hline TN1 & 0.139 \\
TN2 & 0.085 \\
TN3 & 3.059 \\
TN4 & 0.106 \\
TN5 & 0.091 \\
TN6 & 0.064 \\
TN7 & 0.167 \\
TN8 & 0.115 \\
TN9 & 0.130 \\
TN10 & 0.060 \\
TN11 & 0.070 \\
TN12 & 0.133 \\
TN13 & 0.129 \\
TN14 & 0.196 \\
TN15 & 0.102 \\
TN16 & 0.074 \\
TN17 & 0.097 \\
TN18 & 0.118 \\
TN19 & 0.087 \\
TN20 & 0.076 \\
\hline
\end{tabular}

The 60-min incubation step corresponds to the initial "fast step" rate of DPPH• scavenging, involving the one-electron or one-proton transfer processes and conversion into quinones. While this represents the main reaction involved in the radical scavenging process, it is not exhaustive, since a variable number of by-products are produced during this process (some are shown in Figure 2 for (+)-catechin-based molecules), that could further contribute to the radical scavenging activity. The secondary antiradical reactions occur at a later stage, as an increasing number of functional groups within the molecules are involved; this is the reason for the complex kinetic curves and long-term incubation period needed to reach the steady state by using tannins as scavenger molecules. Due to the complex chemical structure of tannins the 1-h incubation time, which is usually recommended in literature for the $\mathrm{DPPH} \bullet$ assay, do not provide a full description if the antiradical activity of these compounds. The radical scavenging clearly continued in the later incubation period until the steady state was reached for all the tannins assayed, regardless of the nature of the extracts.

When considering the overall reaction period and performing periodical comparisons between $\mathrm{DPPH} \bullet$ values and the CV response obtained for tannins, we obtained a good correlation, which 
tended to increase with longer incubation times (data not shown). Best performances were obtained comparing DPPH• and CV results after $24 \mathrm{~h}$ incubation time (steady-state), with satisfactory model fitting parameters ( $\mathrm{R}^{2}$ 0.925; MSE 0.000; RMSE 0.020; standard error 0.065). The DPPH• and CV results obtained at the steady state are listed in Table 3 (Section 2.3).

\subsection{Antioxidant Activity of Tannins}

Combining results obtained by the DPPH• colorimetric assay under steady-state conditions and cyclic voltammetry measurements, information was gained about the redox properties and radical scavenging activity induced by electron and hydrogen-transfer, which are key processes related to the antioxidant capacity of tannins. The anodic peak occurring at around $500 \mathrm{mV}$ in the voltammograms accounted for the oxidation of catechol and pyrogalloyl moieties of polyphenolic compounds. These same moieties react readily with the DPPH• radical itself, a weak oxidizing agent. The ortho -di (catechol) and -tri (pyrogallol) hydroxyl substitutions on flavonoids and benzoic acids increase the antioxidant activity of these compounds, which are similarly captured by the two analytical approaches [14].

Table 3 reports antiradical (\%) and redox (mM CE) activities for the extracts selected in this study. Among them, samples TN2 exhibited a strong antioxidant activity, in agreement with the redox current measured under the $500 \mathrm{mV}$ peaks (TN2:DPPH• $=83.4 \%, \mathrm{CV} 500 \mathrm{mV}=0.365 \mathrm{TN} 14: \mathrm{DPPH} \bullet=74.6 \%$, CV $500 \mathrm{mV}=0.338 \mathrm{mM}$ CE). The sample TN2 is obtained by extracting green tea leaves; despite it belonging to the proanthocyanidin-based tannins group, its reducing properties are improved when compared to the condensed tannins TN5 and TN12, extracted from grapes (TN5: DPPH $\bullet=48.5 \%, \mathrm{CV}$ $500 \mathrm{mV}=0.221$; TN12: DPPH• $=40.9 \%, \mathrm{CV} 500 \mathrm{mV}=0.158 \mathrm{mM} \mathrm{CE}$ ). It was previously observed [26] that galloylation introduced on the flavanol-based compounds increases their effectiveness as radical scavengers, and this structure is likely to occur in proanthocyanidins derived from green tea leaves and grape seeds [27].

Table 3. DPPH• radical scavenging values (\%) at the steady-state ( $24 \mathrm{~h}$ incubation time), and charge passed to $500 \mathrm{mV}$ (converted to $\mathrm{mM}(+)$-catechin equivalent) for the tannins investigated.

\begin{tabular}{|c|c|c|}
\hline Samples & $\begin{array}{c}\text { DPPH• Radical Scavenging }(\%) \text { at } \\
\text { the Steady State }(24 \mathrm{~h})\end{array}$ & $\mathrm{CV}-200 \mathrm{mV}$ to $500 \mathrm{mV}$ \\
\hline Code & $\%$ decrease $\mathrm{Abs}_{517 \mathrm{~nm}}$ & $\mathrm{mMCE}$ \\
\hline TN1 & $49.8 \pm 0.1$ & $0.225 \pm 0.001$ \\
\hline TN2 & $83.4 \pm 0.8$ & $0.365 \pm 0.008$ \\
\hline TN3 & $47.0 \pm 0.3$ & $0.187 \pm 0.006$ \\
\hline $\mathrm{TN} 4$ & $51.5 \pm 1.2$ & $0.258 \pm 0.008$ \\
\hline TN5 & $48.5 \pm 0.1$ & $0.221 \pm 0.008$ \\
\hline TN6 & $69.3 \pm 0.4$ & $0.349 \pm 0.002$ \\
\hline TN7 & $70.9 \pm 1.1$ & $0.335 \pm 0.006$ \\
\hline TN8 & $59.1 \pm 0.1$ & $0.311 \pm 0.002$ \\
\hline TN9 & $63.8 \pm 0.6$ & $0.313 \pm 0.005$ \\
\hline TN10 & $30.2 \pm 1.2$ & $0.115 \pm 0.004$ \\
\hline TN11 & $38.2 \pm 3.2$ & $0.133 \pm 0.007$ \\
\hline TN12 & $40.9 \pm 1.2$ & $0.158 \pm 0.008$ \\
\hline TN13 & $55.0 \pm 0.6$ & $0.234 \pm 0.007$ \\
\hline TN14 & $74.6 \pm 0.1$ & $0.338 \pm 0.006$ \\
\hline TN15 & $53.9 \pm 0.2$ & $0.232 \pm 0.011$ \\
\hline TN16 & $52.0 \pm 0.5$ & $0.233 \pm 0.004$ \\
\hline TN17 & $50.0 \pm 0.1$ & $0.199 \pm 0.003$ \\
\hline TN18 & $62.8 \pm 0.2$ & $0.282 \pm 0.005$ \\
\hline TN19 & $57.0 \pm 0.1$ & $0.237 \pm 0.003$ \\
\hline TN20 & $51.1 \pm 0.1$ & $0.219 \pm 0.003$ \\
\hline
\end{tabular}


The extract TN7, obtained from grape seeds, showed similarity with TN14, extracted from tara and gallnut rich in tannic acid and gallic acid-based structures; their redox and scavenging activity were comparable under the present experimental conditions (TN7: DPPH $\bullet=70.9 \%$, CV $500 \mathrm{mV}=$ $0.335 \mathrm{mM} \mathrm{CE}$; TN14 DPPH $\bullet=74.6 \%$, CV $500 \mathrm{mV}=0.338 \mathrm{mM} \mathrm{CE}$ ), possibly due to their similar composition. In both cases the compounds are characterized by a carboxylic function (gallic acid units in the gallotannin and galloyl and gallate substituents in the grape seed proanthocyanidin), with enhanced antiradical effects $[28,29]$.

The ellagitannins assayed and obtained from American oak (TN10) and French oak (TN11), together with samples TN15, TN16, TN17 and TN20 also extracted from oak with generic geographical origin, are considered key tannins to be exploited in the wine industry, resembling the long-term storage of fine wines in barrels. Nevertheless, TN10 and TN11 showed the lowest radical scavenging (DPPH $\bullet=30.2 \%$ and $38.2 \%$ of radical scavenged, respectively) and redox values (CV $500 \mathrm{mV}=0.115$ and $0.133 \mathrm{mM} \mathrm{CE}$, respectively) of the ellagitannin series (TN15, TN16, TN17 and TN20 average $\mathrm{DPPH} \bullet=51.8 \pm 1.65 \%$; average CV $500 \mathrm{mV}=0.221 \pm 0.0158 \mathrm{mMCE}$ ). The occasional drop in TN10 and TN11 was ascribed to a lower effectiveness of the extraction process and reduced content of bioactive compounds more than to the intrinsic composition of these extracts, this hypothesis requires further confirmation by analyzing the effective polyphenolic content of the extract.

Some blended formulations are included in the sample set and they showed variability in terms of fractions of different tannins added, which resulted in variable scavenging activities and electrochemical redox values observed. The interpretation of results and definition of structure-activity would require more complex analytical techniques for the definition of the compositional profiling of the blends, and the relative contribution of active fraction involved in the antioxidant mechanisms.

The use of tannins in oenology is gaining increasing attention due to their unique technological properties, sensory impact, and nutraceutical value [5,30-32]. Since they can be both sourced from grapes and added as exogenous products during winemaking, a proper understanding of the compositional properties and bioactivity of commercial formulations is a challenging opportunity for winemakers. The antioxidant activity of tannins is considered an opportunity to lower the use of potentially harmful additives, i.e., $\mathrm{SO}_{2}$, and to preserve the shelf-life of wines without altering their sensory perceptions.

\section{Conclusion}

Along with dedicated analytical methods which are exploited in wine research, there is a need for rapid and reliable analytical tools, with simplified instrumentation and data output to obtain technological information and support decisions along the supply chain. This also includes fast methods which provide information about the correlation between the dosage of the commercial products and related bioactivity.

In this work, spectrophotometric (DPPH•) and electrochemical (CV) methods were compared according to their ability to disclose the antioxidant activity of botanical extracts used in oenology for protective purposes. The DPPH• measurements confirmed previous observation about potential limits of this colorimetric assay. The main issue was the long time required to obtain a realistic representation of the antioxidant capacity, which is possibly due to the complex chemical structures involved in the antioxidant reactions, together with the number of by-products and side-reactions contributing to the overall antiradical effect. The CV measures was shown to be well correlated with the DPPH• values obtained at the steady state. Moreover, compositional considerations on the extracts can be derived from the voltammetry profiles.

$\mathrm{CV}$ is a fast analytical approach for the study of the antioxidant capacity of tannin compounds, giving reliable and reproducible results. It does not require special reagent mixtures and long incubation times, and the only reactant required is a supporting electrolyte with a known $\mathrm{pH}$ value, where the sample is dissolved to a suitable concentration. Moreover, only thermodynamic properties of the molecules are involved in the electrochemical measurements, without being affected by further 
properties of the sample, such as color and turbidity, which strongly influence the spectrophotometric assays. Given these considerations, CV is well suited for quality control, providing a fast and reliable method to monitor the redox activity of tannin additives for use in the wine supply chain, to modulate dosages and to improve the protection of wines and musts against oxidation.

\section{Materials and Methods}

\subsection{Tannins}

Twenty tannins commercially available as lyophilized, food-grade powders for oenological use were selected for this case study, accounting for the variability of the botanical sources. General information on their composition is reported in Table 4, as provided by the product suppliers (Enologica Vason, Verona, Italy; HTS Enologia, Marsala, Italy; Laffort, Bordeaux Cedex, FR; AEB Group, Brescia, Italy). Stock solutions of dry tannins were prepared at $1 \mathrm{~g} / \mathrm{L}$ in a model wine solution, and further diluted for analytical purposes. The model wine solution was made up of $12 \%$ v/v ethanol (>99\%) in distilled water, with the addition of L-tartaric acid $0.033 \mathrm{M}$ and $\mathrm{NaOH}$ to reach $\mathrm{pH}$ 3.6. The model wine buffer was prepared using Merck HPLC-grade reagents without further purification (Merck, Darmstadt, Germany). Stock solutions of monomeric standards: (+)-catechin, gallic acid, ellagic acid, were prepared under the same conditions for comparative purposes.

Table 4. Commercial oenological tannins selected for this work with the generic compositional information provided by suppliers. The classification as "Blend "refers to the mixture of tannins from different botanical sources as described in the commercial label.

\begin{tabular}{|c|c|c|}
\hline Code & Chemical Classification & Botanical Origin \\
\hline TN1 & Ellagitannin & White fruits tree wood \\
\hline TN 2 & Proanthocyanidin & Green tea \\
\hline TN 3 & Proanthocyanidin & Unknown \\
\hline TN 4 & Blend, not specified & Unknown \\
\hline TN 5 & Proanthocyanidin & Grape \\
\hline TN 6 & Ellagitannin & Chestnut heartwood \\
\hline TN 7 & Proanthocyanidin & Grape seed \\
\hline TN 8 & Blend: proanthocyanidin/ellagitannins & Unknown \\
\hline TN 9 & $\begin{array}{l}\text { Blend: proanthocyanidin/ } \\
\text { ellagitannin/gallotannin }\end{array}$ & Limousin French oak, tara, gall, green tea \\
\hline TN 10 & Ellagitannin & American oak \\
\hline TN 11 & Ellagitannin & French oak (Allier) \\
\hline TN 12 & Proanthocyanidin & Grape \\
\hline TN 13 & $\begin{array}{l}\text { Blend: proanthocyanidin/ } \\
\text { ellagitannin/gallotannin }\end{array}$ & Limousin French oak, gall, grape \\
\hline TN 14 & Not specified & Unknown \\
\hline TN 15 & Ellagitannin & French oak (Limousin) \\
\hline TN 16 & Ellagitannin & Selected Quercus woods \\
\hline TN 17 & Ellagitannin & French oak \\
\hline TN 18 & Ellagitannin & Red fruit tree wood \\
\hline TN 19 & $\begin{array}{l}\text { Blend: proanthocyanidin/ } \\
\text { ellagitannin/gallotannin }\end{array}$ & Tara, gall, green tea, ellagitannins from oak \\
\hline TN 20 & Ellagitannin & French oak (Allier) \\
\hline
\end{tabular}

\subsection{Chemicals and Reagents}

The 2,2-diphenyl-1-picrylhydrazyl (DPPH•) free radical (95\%) and pure methanol HPLC-grade $(99.8 \%)$ used for the spectrophotometric assay, together with the (+)-catechin monohydrate analytical standard (98\%) used to calibrate the CV measurements, gallic acid and ellagic acid standards, were purchased from Sigma-Aldrich (Sigma-Aldrich, Saint Louis, MO, United States). 


\section{3. $\mathrm{DPPH} \bullet$ Assay}

The DPPH• assay was performed according to the originally proposed method [11] modified by Villaño et al. [10]. Briefly, $100 \mu \mathrm{L}$ of tannin solutions containing $58 \mathrm{mg} / \mathrm{L}$ powders were added to $2.9 \mathrm{~mL}$ of $200 \mu \mathrm{M}$ DPPH• (molar mass: $394.32 \mathrm{~g} / \mathrm{mol}$ ) in methanol. The resulting solutions were vigorously shaken and hermetically sealed to prevent solvent evaporation, incubated in the dark at room temperature and sampled after $15 \mathrm{~min}, 30 \mathrm{~min}, 60 \mathrm{~min}, 180 \mathrm{~min}$ incubation, respectively, until reaching the steady state of reaction $(1440 \mathrm{~min}, 24 \mathrm{~h}$ ). The absorbance was measured at $517 \mathrm{~nm}$ in 10 mm plastic cuvettes against pure methanol, using a Shimadzu UV mini 1240 spectrophotometer (Kyoto, Japan). The results were expressed as percentage of inhibition [30], using the following formula:

$$
\% \text { inhibition }=[(\mathrm{Ab}-\mathrm{As}) / \mathrm{Ab}] \times 100
$$

where: $\mathrm{Ab}=$ absorbance of the reagent blank, and As = absorbance of the sample. Each experiment was run in triplicate and results are provided as average values.

Calculation of the zero-order kinetics related to the fast step of the DPPH• radical scavenging reaction by tannins were performed using the Shimadzu UVProbe software connected to the Shimadzu UV mini 1240 spectrophotometer in the kinetics (time-course) measurement mode (Shimadzu, Kyoto, Japan). The measurements were performed at the wavelength of $517 \mathrm{~nm}$, starting from the mixing of tannins and DPPH• solutions (time zero) and setting a total of 30 subsequent readings separated by time steps of $120 \mathrm{~s}$ (3600 s total time). The resulting absorbance values (a.u.) compared to the elapsed time (s) were used to calculate the kinetic rates; results were expressed as abs $517 \mathrm{~nm} \mathrm{~s}^{-1}$ and are reported in Table 2 (Section 2.2).

\subsection{Cyclic Voltammetry}

The same dilution used in the spectrophotometric experiment was found suitable for CV experiments, performed according to the method previously described [14]. The following equipment was used for CV analysis: A Bioanalytical Systems (BAS) 100A electrochemical analyzer, a BAS C2 electrochemical cell, a $3 \mathrm{~mm}$ glassy carbon disc electrode (working electrode, BAS M-2012), a platinum counter electrode and an $\mathrm{Ag} / \mathrm{AgCl}$ reference electrode (+207 $\mathrm{mV}$ compared to SHE). The glassy carbon electrode was polished with $3 \mu \mathrm{m}$ alumina powder (PK-4 polishing kit), then rinsed with ultrapure $18 \mathrm{M} \Omega$ water, to avoid surface passivation and poisoning. The scan range was taken from $-200 \mathrm{mV}$ to $500 \mathrm{mV}$, in order to record the first anodic peak, related to reactive polyphenols. The voltammograms were recorded at a scan rate of $100 \mathrm{mV} / \mathrm{s}$ and a sensitivity of $1 \mu \mathrm{A}$. A blank run was recorded daily in the model wine solution and was used to subtract the background current. Each experiment was run in triplicate and the average values of the increase in anodic current with potential, $\mu \mathrm{A} \times \mathrm{mV}$ (current integrated) were expressed in $\mathrm{mM}(+)$-Catechin Equivalents (CE), using a calibration curve of the standard over the range of $0.0078 \mathrm{mM}$ to $1.0 \mathrm{mM}$.

\subsection{Data Processing}

Microsoft Excel was used for data entry, whereas derivatives of the cyclic voltammograms were calculated using OriginPro 8 (Origin Lab Corp., Northampton, MA, USA). All analyses were performed in triplicate and results were provided as average values; statistical analyses were performed with the XLSTAT (Addinsoft 2018, Paris, France) software.

Author Contributions: Individual contribution of authors were as follows: A.R., Investigation, writing一original draft preparation; G.P.P., Investigation, resources and data curation; N.T., Validation and formal analysis; A.V. and P.A.K., Methodology, writing-review and editing.

Funding: This research received no external funding.

Acknowledgments: Authors gratefully acknowledge Enologica Vason, HTS Enologia, Laffort and AEB group, for having provided the free samples of commercial tannins. Authors A.R., G.P.P. and A.V. also acknowledge the School of Chemical Sciences (University of Auckland) where they were guest scientists. 
Conflicts of Interest: The authors declare no conflict of interest.

\section{References}

1. Kaur, C.; Kapoor, H.C. Antioxidant activity and total phenolic content of some Asian vegetables. Int. J. Food Sci. Technol. 2002, 37, 153-161. [CrossRef]

2. Vazallo-Valleumbrocio, G.; Medel-Marabolí, M.; Peña-Neira, Á.; López-Solís, R.; Obreque-Slier, E. Commercial enological tannins: Characterization and their relative impact on the phenolic and sensory composition of Carménère wine during bottle aging. LWT-Food Sci. Technol. 2017, 83, 172-183. [CrossRef]

3. Vivas, N.; Bourgeois, G.; Vitry, C.; Glories, Y.; Freitas, V.D. Determination of the composition of commercial tannin extracts by liquid secondary ion mass spectrometry (LSIMS). J. Sci. Food Agric. 1996, 72, 309-317. [CrossRef]

4. Obreque-Slíer, E.; Peña-Neira, A.; López-Solís, R.; Ramírez-Escudero, C.; Zamora-Marín, F. Phenolic characterization of commercial enological tannins. Eur. Food Res. Technol. 2009, 229, 859-866. [CrossRef]

5. Harbertson, J.F.; Parpinello, G.P.; Heymann, H.; Downey, M.O. Impact of exogenous tannin additions on wine chemistry and wine sensory character. Food Chem. 2012, 131, 999-1008. [CrossRef]

6. Versari, A.; Toit, W.; Parpinello, G.P. Oenological tannins: A review. Aust. J. Grape Wine Res. 2013, 19, 1-10. [CrossRef]

7. Ricci, A.; Olejar, K.J.; Parpinello, G.P.; Mattioli, A.U.; Teslić, N.; Kilmartin, P.A.; Versari, A. Antioxidant activity of commercial food grade tannins exemplified in a wine model. Food Addit. Contam. Part. A 2016, 33, 1761-1774. [CrossRef]

8. Waterhouse, A.L.; Laurie, V.F. Oxidation of wine phenolics: A critical evaluation and hypotheses. Am. J. Enol. Vitic. 2006, 57, 306-313.

9. Sànchez-Moreno, C. Review: Methods used to evaluate the free radical scavenging activity in foods and biological systems. Food Sci. Technol. Int. 2002, 8, 121-137. [CrossRef]

10. Villaño, D.; Fernàndez-Pachòn, M.S.; Troncoso, A.M.; Garcìa-Parrilla, M.C. Comparison of antioxidant activity of wine phenolic compounds and metabolites in vitro. Anal. Chim. Acta 2005, 538, 391-398. [CrossRef]

11. Brand-Williams, W.; Cuvelier, M.E.; Berset, C. Use of a free radical method to evaluate antioxidant activity. LWT-Food Sci. Technol. 1995, 28, 25-30. [CrossRef]

12. Mishra, K.; Ojha, H.; Chaudhury, N.K. Estimation of antiradical properties of antioxidants using DPPH assay: A critical review and results. Food Chem. 2012, 130, 1036-1043. [CrossRef]

13. Dawidowicz, A.L.; Wianowska, D.; Olszowy, M. On practical problems in estimation of antioxidant activity of compounds by DPPH method (Problems in estimation of antioxidant activity). Food Chem. 2012, 131, 1037-1043. [CrossRef]

14. Kilmartin, P.A.; Zou, H.; Waterhouse, A.L. A cyclic voltammetry method suitable for characterizing antioxidant properties of wine and wine phenolics. J. Agric. Food Chem. 2001, 49, 1957-1965. [CrossRef]

15. Blasco, A.J.; Gonzàlez, M.A.C.; Escarpa, A. Electrochemical approach for discriminating and measuring predominant flavonoids and phenolic acids using differential pulse voltammetry: Towards an electrochemical index of natural antioxidants. Anal. Chim. Acta 2004, 511, 71-81. [CrossRef]

16. Kilmartin, P.A.; Zou, H.; Waterhouse, A.L. Correlation of wine phenolic composition versus cyclic voltammetry response. Am. J. Enol. Vitic. 2002, 53, 294-302.

17. Gizdavic-Nikolaidis, M.; Travas-Sejdic, J.; Bowmaker, G.A.; Cooney, R.P.; Thompson, C.; Kilmartin, P.A. The antioxidant activity of conducting polymers in biomedical applications. Curr. Appl. Phys. 2004, 4, 347-350. [CrossRef]

18. Cosio, M.S.; Buratti, S.; Mannino, S.; Benedetti, S. Use of an electrochemical method to evaluate the antioxidant activity of herb extracts from the Labiatae family. Food Chem. 2006, 97, 725-731. [CrossRef]

19. Bortolomeazzi, R.; Sebastianutto, N.; Toniolo, R.; Pizzariello, A. Comparative evaluation of the antioxidant capacity of smoke flavouring phenols by crocin bleaching inhibition, DPPH radical scavenging and oxidation potential. Food Chem. 2007, 100, 1481-1489. [CrossRef]

20. Pisoschi, A.M.; Cheregi, M.C.; Danet, A.F. Total antioxidant capacity of some commercial fruit juices: Electrochemical and spectrophotometrical approaches. Molecules 2009, 14, 480-493. [CrossRef] 
21. Cuartero, M.; Ortuño, J.A.; Truchado, P.; Garcìa, M.S.; Tomàs-Barberàn, F.A.; Albero, M.I. Voltammetric behaviour and square-wave voltammetric determination of the potent antioxidant and anticarcinogenic agent ellagic acid in foodstuffs. Food Chem. 2011, 128, 549-554. [CrossRef]

22. Graham, H.N. Green tea composition, consumption, and polyphenol chemistry. Prev. Med. 1992, 21, 334-350. [CrossRef]

23. Pasrija, D.; Anandharamakrishnan, C. Techniques for extraction of green tea polyphenols: A review. Food Bioprocess Technol. 2015, 8, 935-950. [CrossRef]

24. Ricci, A.; Parpinello, G.P.; Palma, A.S.; Teslić, N.; Brilli, C.; Pizzi, A.; Versari, A. Analytical profiling of food-grade extracts from grape (Vitis vinifera sp.) seeds and skins, green tea (Camellia sinensis) leaves and Limousin oak (Quercus robur) heartwood using MALDI-TOF-MS, ICP-MS and spectrophotometric methods. J. Food Compos. Anal. 2017, 59, 95-104. [CrossRef]

25. Ricci, A.; Lagel, M.C.; Parpinello, G.P.; Pizzi, A.; Kilmartin, P.A.; Versari, A. Spectroscopy analysis of phenolic and sugar patterns in a food grade chestnut tannin. Food Chem. 2016, 203, 425-429. [CrossRef]

26. Yokozawa, T.; Chen, C.P.; Dong, E.; Tanaka, T.; Nonaka, G.-I.; Nishioka, I. Study on the inhibitory effect of tannins and flavonoids against the 1,1-diphenyl-2-picrylhydrazyl radical. Biochem. Pharmacol. 1998, 56, 213-222. [CrossRef]

27. de Freitas, V.A.; Glories, Y.; Bourgeois, G.; Vitry, C. Characterisation of oligomeric and polymeric procyanidins from grape seeds by liquid secondary ion mass spectrometry. Phytochemistry 1998, 49, 1435-1441. [CrossRef]

28. de Gaulejac, N.S.C.; Vivas, N.; de Freitas, V.; Bourgeois, G. The influence of various phenolic compounds on scavenging activity assessed by an enzymatic method. J. Sci. Food Agric. 1999, 79, 1081-1090. [CrossRef]

29. Salah, N.; Miller, N.J.; Paganga, G.; Tijburg, L.; Bolwell, G.P.; Rice-Evans, C. Polyphenolic flavanols as scavengers of aqueous phase radicals and as chain-breaking antioxidants. Arch. Biochem. Biophys. 1995, 322, 339-346. [CrossRef]

30. Dudonné, S.; Vitrac, X.; Coutière, P.; Woillez, M.; Mérillon, J.-M. Comparative study of antioxidant properties and total phenolic content of 30 plant extracts of industrial interest using DPPH, ABTS, FRAP, SOD, and ORAC assays. J. Agric. Food Chem. 2009, 57, 1768-1774. [CrossRef]

31. Soares, S.; Sousa, A.; Mateus, N.; de Freitas, V. Effect of condensed tannins addition on the astringency of red wines. Chem. Senses 2011, 37, 191-198. [CrossRef]

32. Picariello, L.; Gambuti, A.; Picariello, B.; Moio, L. Evolution of pigments, tannins and acetaldehyde during forced oxidation of red wine: Effect of tannins addition. LWT-Food Sci. Technol. 2017, 77, 370-375. [CrossRef]

Sample Availability: Samples of the compounds are commercially available oenological tannins; analytical samples are available from the Authors G.P.P., A.R. and A.V. 